MAY A; CECÍLIO FILHO AB; PORTO DRQ; VARGAS PF; BARBOSA JC. 2007. Produtividade de híbridos de cebola em função da população de plantas e da fertilização nitrogenada e potássica. Horticultura Brasileira 25: 053-059.

\title{
Produtividade de híbridos de cebola em função da população de plantas e da fertilização nitrogenada e potássica
}

\author{
André Mayi ; Arthur Bernardes Cecílio Filho²; Diego Resende de Queirós Porto ${ }^{2}$; Pablo Forlan Vargas²; \\ José Carlos Barbosa ${ }^{2}$ \\ ${ }^{1}$ IAC, Av. Barão de Itapura, 1481, C. Postal 28, 13001-970 Campinas-SP; ${ }^{2}$ UNESP/FCAV,Via de acesso Prof. Paulo D. Castellane s/n, \\ 14884-900 Jaboticabal-SP; E-mail: amay@iac.sp.gov.br; rutra@fcav.unesp.br.
}

\section{RESUMO}

O trabalho foi realizado em São José do Rio Pardo-SP, de 07 de março a 08 de agosto de 2004 para avaliar o efeito do nitrogênio, potássio e população de plantas na massa do bulbo e produtividade de duas cultivares de cebola. O delineamento experimental utilizado foi de blocos ao acaso, com 4 repetições, com os tratamentos em esquema fatorial 2 × 4 × 4 × 4: cultivares (Optima e Superex), doses de nitrogênio $\left(0 ; 50 ; 100\right.$ e $150 \mathrm{~kg} \mathrm{ha}^{-1}$ de $\left.\mathrm{N}\right)$, doses de potássio $(0$; $75 ; 150$ e $225 \mathrm{~kg} \mathrm{ha}^{-1}$ de $\mathrm{K}_{2} \mathrm{O}$ ) e população de plantas (60; 76; $92 \mathrm{e}$ $108 \mathrm{pl} \mathrm{m}^{-2}$ ). A produtividade foi influenciada pela interação doses de $\mathrm{N}$ e cultivar e doses de $\mathrm{K}$ e cultivar, enquanto a massa de bulbo, além das interações verificadas para a produtividade, foi influenciada significativamente pela interação $\mathrm{N}$ e população de plantas. Não houve interação tripla para nenhuma das variáveis estudadas. Massas menores de bulbo foram obtidas com aumento na população de plantas e para todas as populações. $\mathrm{O}$ aumento de $\mathrm{N}$ proporcionou incremento na massa do bulbo. Para se obter $90 \%$ da produtividade máxima, estimada em $71 \mathrm{t} \mathrm{ha}^{-1}$ e $64,8 \mathrm{tha}^{-1}$ para as cultivares Superex e Optima, em solo semelhante ao do presente estudo, devem ser fornecidas quantidades de $125 \mathrm{~kg} \mathrm{ha}^{-1}$ e $105 \mathrm{~kg} \mathrm{ha}^{-1}$ de $\mathrm{N}$, respectivamente, sem a necessidade de aplicação de $\mathrm{K}$.

Palavras-chave: Allium cepa L., nutrição, produção, densidade de plantas.

\begin{abstract}
Plant density and nitrogen and potassium fertilization rates on yield of onion hybrids

An experiment was carried out in Sao Jose do Rio Pardo, the onion producing area in São Paulo State, Brazil,from March 07 to August 08, 2004. The objective of the research was to evaluate the effects of nitrogen and potassium fertilizer rates on yield of two onion cultivars, growing in different plant populations. The experimental design was in blocks with four replications in a $2 \times 4 \times 4 \times 4$ factorial cheme: cultivars (Optima and Superex), nitrogen $(0 ; 50 ; 100$ and $150 \mathrm{~kg} \mathrm{ha}^{-1}$ of $\left.\mathrm{N}\right)$, potassium $\left(0 ; 75 ; 150\right.$ and $225 \mathrm{~kg} \mathrm{ha}^{-1}$ of $\left.\mathrm{K}_{2} \mathrm{O}\right)$ and plant population $\left(60 ; 76 ; 92\right.$ and $\left.108 \mathrm{pl} \mathrm{m}^{-2}\right)$. The effects of $\mathrm{N}$ and $\mathrm{K}$ doses on yield were cultivar dependent. Average individual bulb mass increased with decreasing plant population and increasing nitrogen doses application. To reach $90 \%$ of the maximum expected crop yield of $71 \mathrm{t} \mathrm{ha}^{-1}$ for 'Superex' and 64,8 $\mathrm{tha}^{-1}$ for 'Optima', nitrogen doses of 125 and $105 \mathrm{~kg} \mathrm{ha}^{-1}$ should be applied, respectively, without potassium fertilization.
\end{abstract}

Keywords: Allium cepa L., nutrition, yield, plant density.

(Recebido para publicação em 28 de janeiro de 2006; aceito em 9 de abril de 2007)

$\mathrm{N}$ Brasil, a cebola é considerada a terceira hortaliça mais importante em termos de valor econômico, ficando atrás apenas da batata e do tomate. É preferencialmente consumida in natura em saladas, temperos e condimentos (Boeing, 2002).

O munícipio de São José do Rio Pardo é uma tradicional zona produtora de cebola, cujo sistema de cultivo predominante é o transplantio de mudas, com produtividades que dificilmente passam de $50 \mathrm{t} \mathrm{ha}^{-1}$ de bulbos, mesmo nos melhores anos de cultivo ${ }^{1}$.

A competição por água, luz e nutrientes faz com que o tamanho dos bulbos e a produtividade total variem con- forme a população de plantas na área (Nichols, 1967). Stoffella (1996) observou que com o aumento da população de plantas, alterando-se o espaçamento na linha e entre as plantas $(0,229$ a 0,457 m entre linhas x 0,076 a 0,229 m entre plantas), houve aumento da produtividade de bulbos de cebola, acompanhado de redução da massa do bulbo.

Santos et al. (2000) avaliaram o comportamento da cv. Texas Grano em diferentes espaçamentos $(0,05 ; 0,10 \mathrm{e}$ 0,15 m entre plantas na linha), utilizando $0,3 \mathrm{~m}$ nas entrelinhas. Os autores observaram que os menores espaçamentos promoveram reduções na massa do bulbo, com grande influência na produtividade. $\mathrm{O}$ menor espaçamento entre as plantas na linha proporcionou a maior produtividade (40,3 t de bulbos e $53,1 \mathrm{~g}$ por bulbo), enquanto com 0,10 $\mathrm{m}$ entre plantas obtiveram-se bulbos maiores $(83,4 \mathrm{~g})$, havendo porém redução na produtividade em 19,35\%. Dellacecca \& Lovato (2000), estudando três populações de plantas $(26,6 ; 40$ e 80 plantas $\mathrm{m}^{-2}$ ), verificaram que a maior população proporcionou maior produtividade (31 t ha-1), embora com menor massa do bulbo, sendo 105,5 g e $189,0 \mathrm{~g}$ por bulbo para a maior e menor densidade de plantas, respectivamente. Rumpel \& Felczynski (2000) estudaram o efeito da população de plantas entre

\footnotetext{
${ }^{1}$ Breda Junior, J. M. (Cooperativa dos Cafeicultures de Guaxupé
}

- Filial São José do Rio Pardo) Informação Pessoal, 2005. 
20 e 140 plantas $\mathrm{m}^{-2}$ na produtividade da cultura de cebola e verificaram que as maiores produtividades foram de 32,8 e 59,0 t ha $^{-1}$ respectivamente no primeiro e no segundo ano, obtidas com 80 plantas $\mathrm{m}^{-2}$.

VIDIGAL et al. (2002) relatam, para a 'Alfa Tropical', que a planta toda extraiu 124,$62 ; 21,35 ; 130,73 ; 62,78 ; 8,47$; 20,14 kg ha-1 de N, P, K, Ca, Mg e S, respectivamente.

Henriksen (1987), avaliando doses de $\mathrm{N}$ entre 0 e $180 \mathrm{~kg} \mathrm{ha}^{-1}$, observou que $120 \mathrm{~kg} \mathrm{ha}^{-1}$ de $\mathrm{N}$, utilizando o fertilizante nitrato de cálcio, proporcionou produtividade de 61,5 $\mathrm{t} \mathrm{ha}^{-1}$ de bulbos comerciais em cultura estabelecida pelo sistema de transplantio na população de 80 plantas $\mathrm{m}^{-2}$. Em solos da região do Cerrado é comum a aplicação de doses em torno de $120 \mathrm{~kg} \mathrm{ha}^{-1}$ de $\mathrm{N}$ (Magalhães, 1993).

A exigência de potássio pela cebola é alta, sendo um nutriente de grande importância no transporte de fotoassimilados das folhas para os orgãos de reserva (Faquin, 1994). Apesar da cebola extrair grandes quantidades de potássio, as respostas da cultura a este nutriente são pequenas (Magalhães, 1993), de forma contrária ao que ocorre com o N (Brewster, 1994). Araújo \& Costa (1975), em solo de cerrado, não observaram influência da variação no fornecimento do potássio entre 0 e 90 $\mathrm{kg} \mathrm{ha}^{-1}$ de $\mathrm{K}_{2} \mathrm{O}$, na produtividade de cebola. A ausência de resposta do $\mathrm{K}$ na produtividade da cebola também foi observada por Pande \& Mundra (1971), utilizando até $89,6 \mathrm{~kg} \mathrm{ha}^{-1}$ de $\mathrm{K}_{2} \mathrm{O}$.

$\mathrm{O}$ trabalho objetivou quantificar a produtividade de cultivares de cebola, em função da população de plantas e da fertilização com nitrogênio e potássio em cultura estabelecida por semeadura direta.

\section{MATERIAL E MÉTODOS}

O experimento foi conduzido no município de São José do Rio PardoSP. O delineamento experimental utilizado foi de blocos ao acaso, com os tratamentos em esquema fatorial $2 \times 4$ x 4 x 4, com quatro repetições. Os fatores avaliados foram: duas cultivares (híbridos Optima e Superex); quatro populações de plantas (60; 76; 92 e 108 plantas $\left.\mathrm{m}^{-2}\right)$; quatro doses de nitrogênio $\left(0 ; 50 ; 100\right.$ e $150 \mathrm{~kg} \mathrm{ha}^{-1}$ de N) e 4 doses de potássio $\left(0 ; 75 ; 150\right.$ e $225 \mathrm{~kg} \mathrm{ha}^{-1}$ de $\mathrm{K}_{2} \mathrm{O}$ ). As parcelas foram localizadas em canteiros de 1,2 m de largura, com cinco linhas longitudinais ao canteiro e com comprimento de $2,5 \mathrm{~m}$. O espaçamento entre linhas foi de $0,275 \mathrm{~m}$.

O solo do local do experimento é classificado como um LATOSSOLO VERMELHO AMARELO, textura argilosa (Embrapa, 1999). A análise do solo na profundidade de 0 a $0,2 \mathrm{~m}$ revelou $\mathrm{pH}$ em $\mathrm{CaCl}_{2}$ de 5,2 e $25 \mathrm{~g} \mathrm{dm}^{-3}$ de matéria orgânica, além de $24 \mathrm{mg} \mathrm{dm}^{-3}$ de P-resina. Os teores de $\mathrm{K}, \mathrm{Ca}, \mathrm{Mg}$, $\mathrm{H}+\mathrm{Al}$ foram, respectivamente, 1,$4 ; 31$; 10 e $28 \mathrm{mmol}_{\mathrm{c}} \mathrm{dm}^{-3}$ e a CTC efetiva igual a 70,4 mmol $\mathrm{dm}^{-3}$, segundo metodologia descrita por Raij et al. (2001).

Com base na análise de solo feita em laboratório da UNESP em Jaboticabal e nas recomendações de Trani et al. (1996) para a calagem e adubação da cultura da cebola, sistema de transplantio, fez-se aplicação de calcário, um mês antes da semeadura, para elevar a saturação por bases a $70 \%$. Na semana da instalação do experimento, foram aplicados $300 \mathrm{~kg} \mathrm{ha}^{-1}$ de $\mathrm{P}_{2} \mathrm{O}_{5}$, utilizando como fonte superfosfato simples, à lanço em área total, e 1,5 kg ha-1 de B, utilizando como fonte bórax.

Dois dias antes da semeadura foram aplicados $30 \mathrm{~kg} \mathrm{ha}^{-1}$ de $\mathrm{Ne} 40 \mathrm{~kg} \mathrm{ha}^{-1}$ de $\mathrm{K}_{2} \mathrm{O}$ em todos os tratamentos, exceto na testemunha, incorporados com enxada. $\mathrm{O}$ restante do nitrogênio e do potássio de cada tratamento foi parcelado em quantidades iguais e aplicado aos 30, 45 e 60 dias após a semeadura (DAS). Foram empregados como fontes os fertilizantes nitrato de amônio e cloreto de potássio.

A semeadura foi realizada de 7 a 9 de março de 2004. Para auxiliar na implantação do fator população de plantas, a semeadura foi feita em pequenas covas de $3 \mathrm{~cm}$ de profundidade, abertas com o auxílio de um quadro furador metálico. Este furador era composto por um quadro de metal com 1,10 m de largura com cinco barras de ferro soldadas, distanciadas em 27,5 cm entre cada uma delas (espaçamento entre linhas). Em cada barra de ferro foram soldados ferros 3/12" de $3 \mathrm{~cm}$ de comprimento para marcação das covas, distanciados em 6,$92 ; 5,46 ; 4,51 ; 3,84 \mathrm{~cm}$ para a população de planta de 60; 76; 92 e 108 pl $\mathrm{m}^{-2}$. Em cada cova aberta foram colocados cerca de cinco a sete sementes de cebola, sendo realizado um posterior desbaste para estabelecer o devido espaçamento entre plantas para cada parcela. Durante a semeadura, manteve-se o solo umedecido para que as covas permanecessem abertas. Após a semeadura, as covas foram cobertas com terra do próprio canteiro de forma a manter o canteiro uniforme em seu nível. A emergência das plântulas iniciou em 17/03/04 e, aproximadamente 15 dias após, fez-se o desbaste para estabelecer o número correto de plantas em cada parcela, de acordo com o fator população de plantas.

A aplicação de herbicidas, fungicidas e inseticidas foi feita de acordo com as necessidades apresentadas pela lavoura. As irrigações foram diárias durante as primeiras semanas após a semeadura, e a cada quatro dias em complementação às precipitações pluviais, nos períodos subseqüentes, sendo utilizado o sistema de aspersão convencional, com a aplicação de uma lâmina média de $10 \mathrm{~mm}$. O controle de plantas daninhas foi feito com auxílio de enxada sempre que necessário.

$\mathrm{O}$ ponto de colheita foi determinado quando $70 \%$ das plantas "estalaram", ponto este caracterizado pelo amolecimento do pseudocaule e tombamento da parte aérea, baseado na proposta de Finger \& Casali (2002) de 50 a $80 \%$ de estalo. As plantas foram arrancadas e deixadas sobre os canteiros com as folhas distribuídas sobre os bulbos para que ficassem protegidos da irradiação direta. A colheita foi iniciada em 27/07/ 04 e finalizada em 08/08/04. Após quatro dias de insolação, os bulbos foram retirados do local e encaminhados para o laboratório, onde foram feitas as avaliações subseqüentes, após a eliminação da parte aérea e das raízes. As parcelas foram colhidas separadamente, conforme a identificação do ponto de colheita de cada uma delas.

A massa do bulbo ( $\mathrm{g}$ bulbo $^{-1}$ ) foi calculada pela divisão da massa total de bulbos por parcela pelo número de bul- 
Tabela 1. Análise da superfície de resposta para as variáveis: massa do bulbo em função das doses de N e K para a cultivar Optima para 60 $\mathrm{pl} \mathrm{m} \mathrm{m}^{-2}$ (1), para $76 \mathrm{pl} \mathrm{m}$ (2), para $92 \mathrm{pl} \mathrm{m}^{-2}$ (3) e $108 \mathrm{pl} \mathrm{m}^{-2}$ (4); massa do bulbo em função das doses de N e K para a cultivar Superex para $60 \mathrm{pl} \mathrm{m}^{-2}$ (5), para $76 \mathrm{pl} \mathrm{m}^{-2}$ (6), para $92 \mathrm{pl} \mathrm{m}^{-2}$ (7) e $108 \mathrm{pl} \mathrm{m}^{-2}$ (8) e massa do bulbo em função da dose de nitrogênio e da população de plantas de cebola para a cultivar Optima (9) e para a Superex (10) (Analysis of answer to the values: bulb mass as result of doses of $\mathrm{N}$ and $\mathrm{K}$ for the cultivar Optima of $60 \mathrm{pl} \mathrm{m}^{-2}(1)$, of $76 \mathrm{pl} \mathrm{m}^{-2}$ (2), of $92 \mathrm{pl} \mathrm{m}^{-2}$ (3) and $108 \mathrm{pl} \mathrm{m}^{-2}$ (4); bulb mass as result of doses of $\mathrm{N}$ and $\mathrm{K}$ for the cultivar Superex of $60 \mathrm{pl} \mathrm{m}^{-2}$ (5), of $76 \mathrm{pl} \mathrm{m}^{-2}$ (6), of $92 \mathrm{pl} \mathrm{m}^{-2}$ (7) and $108 \mathrm{pl} \mathrm{m}^{-2}$ (8) and, bulb mass as a function of nitrogen doses and of onion plants population for the cultivar Optima (9) and Superex (10)). Jaboticabal, UNESP, 2004.

\begin{tabular}{|c|c|c|c|c|c|c|c|c|c|c|c|}
\hline $\begin{array}{l}\text { Parâme- } \\
\text { tros do } \\
\text { modelo }\end{array}$ & 1 & 2 & 3 & 4 & 5 & 6 & 7 & 8 & $\begin{array}{l}\text { Parâme- } \\
\text { tros do } \\
\text { modelo }\end{array}$ & 9 & 10 \\
\hline $\begin{array}{l}\mathrm{b}_{0} \text { (Inter- } \\
\text { cepto) }\end{array}$ & 69,556100 & 58,602700 & 40,712050 & 32,133800 & 37,823750 & 37,810600 & 29,849400 & 26,968650 & $\begin{array}{l}\mathrm{b}_{0} \text { (Inter- } \\
\text { cepto) }\end{array}$ & 206,930572 & 119,939620 \\
\hline$b_{1}(N)$ & 1,190962 & 0,807842 & 0,802161 & 0,870751 & 1,158478 & 1,190192 & 1,167338 & 0,864746 & $b_{1}(N)$ & 1,111503 & 1,645411 \\
\hline$b_{2}(K)$ & 0,100075 & 0,027284 & 0,177824 & 0,131461 & 0,568172 & 0,221341 & 0,325365 & 0,205197 & $b_{2}(P P)$ & $-2,754748$ & $-1,136735$ \\
\hline$b_{3}(N 2)$ & $-0,003867$ & $-0,002302$ & $-0,002147$ & $-0,003115$ & $-0,002003$ & $-0,003450$ & $-0,003515$ & $-0,002931$ & $b_{3}(\mathrm{~N} 2)$ & $-0,002858$ & $-0,002975$ \\
\hline$b_{4}(N \times K)$ & $-0,000530$ & $-0,000386$ & 0,000084747 & $-0,000144$ & $-0,000244$ & $-0,000823$ & $-0,000342$ & 0,000251 & $b_{4}(N x P P)$ & $-0,002631$ & $-0,006938$ \\
\hline $\mathrm{b}_{5}\left(\mathrm{~K}^{2}\right)$ & $-0,000141$ & 0,000377 & $-0,000657$ & $-0,000257$ & $-0,001899$ & $-0,000328$ & $-0,001138$ & $-0,000665$ & $b_{5}\left(P^{2}\right)$ & 0,011343 & 0,003518 \\
\hline $\begin{array}{l}\text { Teste F } \\
\text { para o } \\
\text { modelo }\end{array}$ & $13,834^{* *}$ & $10,860^{* *}$ & $18,550^{* *}$ & $9,831^{* *}$ & $61,027^{* *}$ & $16,06^{\star *}$ & $63,314^{* *}$ & $18,155^{* *}$ & $\begin{array}{l}\text { Teste F } \\
\text { para o } \\
\text { modelo }\end{array}$ & $113,8^{* *}$ & $163,6^{* *}$ \\
\hline $\mathrm{R}^{2}$ & 0,87 & 0,84 & 0,90 & 0,83 & 0,97 & 0,89 & 0,97 & 0,90 & $\mathrm{R}^{2}$ & 0,98 & 0,99 \\
\hline CV (\%) & 12,11 & 12,90 & 13,08 & 17,15 & 8,52 & 14,24 & 8,16 & 14,69 & CV $(\%)$ & 5,47 & 5,47 \\
\hline $\begin{array}{l}\text { Ponto } \\
\text { crítico N }\end{array}$ & 148,8359 & 171,1212 & 189,6784 & 134,7496 & 281,1417 & 155,5435 & 160,2687 & 155,3568 & $\begin{array}{l}\text { Ponto } \\
\text { crítico N }\end{array}$ & 131,5456 & 41,0015 \\
\hline $\begin{array}{l}\text { Ponto } \\
\text { crítico K }\end{array}$ & 75,2210 & 51,5260 & 147,5886 & 218,3483 & 131,5611 & 142,1310 & 118,8472 & 183,4691 & $\begin{array}{l}\text { Ponto } \\
\text { crítico PP }\end{array}$ & 136,6867 & 201,9886 \\
\hline Tipo & Máximo & $\begin{array}{l}\text { Ponto de } \\
\text { sela }\end{array}$ & Máximo & Máximo & Máximo & Máximo & Máximo & Máximo & Tipo & $\begin{array}{l}\text { Ponto de } \\
\text { sela }\end{array}$ & $\begin{array}{l}\text { Ponto de } \\
\text { sela }\end{array}$ \\
\hline $\begin{array}{l}\text { Valor } \\
\text { predito } \\
\text { no ponto } \\
\text { crítico }\end{array}$ & 161,9489 & 128,4250 & 129,9107 & 105,1526 & 238,0465 & 146,1037 & 142,7277 & 112,9644 & $\begin{array}{l}\text { Valor } \\
\text { predito } \\
\text { no ponto } \\
\text { crítico }\end{array}$ & 91,7685 & 38,8680 \\
\hline
\end{tabular}

**Significativo a $1 \%$ de probabilidade pelo Teste F. (significant at $1 \%$ probability, test $\mathrm{F}$ )

bos colhidos. A estimativa da produtividade $\left(\mathrm{t} \mathrm{ha}^{-1}\right)$ foi realizada a partir da pesagem dos bulbos colhidos das três linhas centrais da parcela (deixando-se uma linha de bordadura de cada lado) em um metro linear de canteiro e feita a proporção para 1 hectare com $5.900 \mathrm{~m}^{2}$ efetivamente cultivados.

Foram utilizados os programas SAS e ESTAT para as análises estatísticas, utilizando-se os dados originais; e programa Statistica, para ajuste das equações polinomiais múltiplas. As equações que regem as regressões polinomiais múltiplas nas superfícies de resposta de segunda ordem seguiram o modelo:

$\mathrm{Y}=\mathrm{b}_{0}+\mathrm{b}_{1}($ Fator 1$)+\mathrm{b}_{2}($ Fator 2$)+$ $\mathrm{b}_{3}(\text { Fator } 1)^{2}+\mathrm{b}_{4}($ Fator 1$) *($ Fator 2$)+$ $\mathrm{b}_{5}(\text { Fator } 2)^{2}$

\section{RESULTADOS E DISCUSSÃO}

Foram constatadas interações significativas entre cultivares e doses de nitrogênio, cultivares e doses de potássio e doses de nitrogênio e população de plantas. Houve efeitos significativos para todos os fatores isoladamente, exceto para cultivares, sobre a massa do bulbo.

Os ajustes de superfícies de resposta de segunda ordem para a massa do bulbo, em todas as populações de plantas, para cada cultivar e para a produtividade são apresentados nas Tabela 1 e 2, respectivamente.

Foram feitas isolinhas combinando $\mathrm{N}$ e K para massa do bulbo para cada população de plantas e para cada cultivar estudada, pois a população de plantas foi muito importante para a expressão da variável massa do bulbo, já que foi verificada interação entre doses de $\mathrm{N}$ e população de plantas e interação entre cultivares e doses de $\mathrm{N}$ e cultivares e doses de $\mathrm{K}$, demonstrando que houve um comportamento diferente para cada cultivar para a expressão dessa variável.

Nas interações, a 'Superex' sempre apresentou massa do bulbo maior que a
'Optima', em todas as populações de plantas, sendo de 188,81 (Figura 1E), 136,17 (Figura 1F), 132,07 (Figura 1G) e $105,05 \mathrm{~g}^{\text {bulbo }}{ }^{-1}$ (Figura $\left.1 \mathrm{H}\right)$, respectivamente com 60; 76; 92 e $108 \mathrm{pl} \mathrm{m}^{-2}$; enquanto para a cultivar Optima, os valores máximos obtidos foram de 153,54 (Figura 1A), 132,74 (Figura 1B), 118,73 (Figura 1C) e 98,44 g bulbo-1 (Figura 1D), respectivamente com 60; 76; 92 e $108 \mathrm{pl} \mathrm{m}^{-2}$.

Observa-se que quanto maior a população de plantas, maiores quantidades de $\mathrm{N}$ foram necessárias para manter a massa do bulbo nos máximos valores, para cada cultivar estudada.

Para a 'Optima', as doses acima de $150 \mathrm{~kg} \mathrm{ha}^{-1}$ de $\mathrm{N}$ com $60 \mathrm{~kg} \mathrm{ha}^{-1}$ de $\mathrm{K}_{2} \mathrm{O}$ não foram suficientes para a produção de bulbos com massa superior a 98,44 g, quando cultivadas com $108 \mathrm{pl} \mathrm{m}^{-2}$ (Figura 1D). Já para 'Superex' (Figura 1H), a aplicação de $150 \mathrm{~kg} \mathrm{ha}^{-1}$ de N com 75 $\mathrm{kg} \mathrm{ha}^{-1} \mathrm{de}_{2} \mathrm{O}$ proporcionou bulbos com massa $105,5 \mathrm{~g}$, na maior população de 
Tabela 2. Análise da superfície de resposta para a variável produtividade em função das doses de N e K para a cultivar Optima (11) e Superex (12) (Analysis of the response for the yield as result of N and K doses for the cultivar Optima (11) and Superex (12)) Jaboticabal, UNESP, 2004.

\begin{tabular}{llcc}
\hline Parâmetros do modelo & Variável & $\mathbf{1 1}$ & $\mathbf{1 2}$ \\
\hline $\mathrm{b}_{0}$ & Intercepto & 26,569500 & 18,190600 \\
$\mathrm{~b}_{1}$ & $\mathrm{~N}$ & 0,547780 & 0,663310 \\
$\mathrm{~b}_{2}$ & $\mathrm{~K}$ & 0,059140 & 0,162223 \\
$\mathrm{~b}_{3}$ & $\mathrm{~N}^{2}$ & $-0,001756$ & $-0,001916$ \\
$\mathrm{~b}_{4}$ & $\mathrm{NxK}$ & $-0,000064000$ & $-0,000143$ \\
$\mathrm{~b}_{5}$ & $\mathrm{~K}^{2}$ & $-0,000075111$ & $-0,000476$ \\
Teste F para o modelo & & $69,719^{* *}$ & $116,64^{* *}$ \\
$\mathrm{R}^{2}$ & & 0,97 & 0,98 \\
\hline $\mathrm{CV}(\%)$ & & 6,18 & 5,96 \\
Ponto crítico N & & 149,9639 & 167,6581 \\
Ponto crítico K & & 329,7934 & 145,2687 \\
Tipo & & Máximo & Máximo \\
Valor predito no ponto crítico & & 77,3951 & 85,5782 \\
\hline
\end{tabular}

**Significativo a $1 \%$ de probabilidade pelo Teste $\mathrm{F}$ (significant at $1 \%$ probability, test $\mathrm{F}$ )

plantas estudada, demonstrando maior resposta da planta às fertilizações.

As duas cultivares apresentaram superfícies de resposta semelhantes à interação doses de $\mathrm{N}$ e populações de plantas. Para a 'Optima', com a elevação das doses de $104 \mathrm{~kg} \mathrm{ha}^{-1}$ até $150 \mathrm{~kg}$ ha ${ }^{-1}$ de $\mathrm{N}$, a massa do bulbo não foi alte-

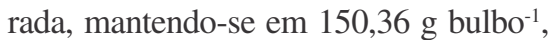
para populações entre 60 e $66 \mathrm{pl} \mathrm{m}^{-2}\left(\mathrm{Fi}^{-}\right.$ gura 2A). Para 'Superex', de 120 a 150 $\mathrm{kg} \mathrm{ha}^{-1}$ de N, a massa do bulbo permaneceu a mesma (168,78 $\mathrm{g}$ bulbo $\left.^{-1}\right)$ com população entre 60 e $67 \mathrm{pl} \mathrm{m}^{-2}$ (Figura 2B).

Para a cultivar Optima, a população de plantas de $60 \mathrm{pl} \mathrm{m}^{-2}$, com aplicação de doses de $\mathrm{N}$ acima de $100 \mathrm{~kg} \mathrm{ha}^{-1}$ associada com até $225 \mathrm{~kg} \mathrm{ha}^{-1}$ de $\mathrm{K}_{2} \mathrm{O}$ ou $110 \mathrm{~kg} \mathrm{ha}^{-1}$ de N sem a aplicação de K, proporciou bulbos com massa de 153,54 g (Figura 1A). Para a 'Superex', a aplicação de $\mathrm{N}$ acima de $124 \mathrm{~kg} \mathrm{ha}^{-1}$ de $\mathrm{N}$, combinada com inúmeras doses de $\mathrm{K}_{2} \mathrm{O}$, proporcionou bulbos com 188,81 g, na menor população de plantas (Figura 1E), demonstrando diferença de desempenho de cada cultivar em diferentes combinações de fertilização e população de plantas. Assim, para que se aumente a população de plantas é preciso associar a fertilização nitrogenada de modo a alcançar maiores massas do bulbo, dentro dos níveis estudados, uma vez que os pontos críticos estão fora da faixa estudada para cada um dos fatores, sendo $131 \mathrm{~kg} \mathrm{ha}^{-1}$ de $\mathrm{N}$ e $137 \mathrm{pl} \mathrm{m}^{-2}$ para
'Optima' e $41 \mathrm{~kg} \mathrm{ha}^{-1}$ de N e 202 pl m-2, para 'Superex' (Figura 2 A e B).

As maiores massas do bulbo, 150,36 e $168,78 \mathrm{~g} \mathrm{bulbo}^{-1}$, respectivamente para a 'Optima' e 'Superex', foram obtidas

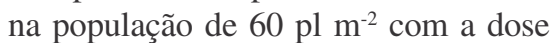
de $150 \mathrm{~kg} \mathrm{ha}^{-1}$ de N (Figura 2A e B).

Para a produção de bulbos com 150 g, preferida pelo mercado consumidor (Silva et al., 1991), considerando-se as doses de 105 e $125 \mathrm{~kg} \mathrm{ha}^{-1}$ de $\mathrm{N}$ para obter as produtividades de 64,8 e $71 \mathrm{t}$ ha $^{-1}$ (90\% da máxima) respectivamente para as cultivares Optima e Superex, foram necessárias $66 \mathrm{pl} \mathrm{\textrm {m } ^ { - 2 }}$ para a 'Optima' e $76 \mathrm{pl} \mathrm{m}{ }^{-2}$ para a 'Superex' (Figura 2C e D), demonstrando a superioridade produtiva da cultivar Superex com aplicação de $\mathrm{N}$, sendo capaz de manter altas produtividades em populações de plantas superiores àquelas limites para a 'Optima', fixando-se a massa do bulbo.

Santos et al. (2000) verificaram também uma redução significativa na massa do bulbo com o aumento do número de plantas por área. No entanto, os autores encontraram reflexos negativos na produtividade quando o espaçamento entre plantas foi aumentado. Houve incremento na massa do bulbo de até $57 \%$, associado a uma queda de $19,35 \%$ na produtividade total, quando o espaçamento entre plantas passou de $0,05 \mathrm{~m}$ para $0,10 \mathrm{~m}$, com $0,3 \mathrm{~m}$ nas entrelinhas.
Houve efeito significativo de doses de nitrogênio e de doses de potássio isoladamente, não havendo efeito de cultivares e da população de plantas sobre a produtividade da cebola. Também foram constatadas interações significativas entre cultivares e doses de nitrogênio e cultivares e doses de potássio.

Em decorrência da interação significativa de cultivares com doses de $\mathrm{N}$ e de $\mathrm{K}$, foram estimadas superfícies de respostas para melhor avaliar a influência do $\mathrm{N}$ e $\mathrm{K}$ na produtividade de cada cultivar (Tabelas 2).

Para as duas cultivares, o efeito da aplicação de nitrogênio foi mais expressivo do que o promovido pelo potássio, verificado pela inclinação da isolinha, que se acentua à medida que se aumenta a dose de N (Figura 2C e D). Considerando a isolinha de produtividade de 68,61 t ha-1 de bulbos para a 'Optima', seria suficiente a aplicação de $100 \mathrm{~kg}$ $\mathrm{ha}^{-1}$ de $\mathrm{N}$ e $108 \mathrm{~kg} \mathrm{ha}^{-1}$ de $\mathrm{K}_{2} \mathrm{O}$. No entanto, para se alcançar à próxima isolinha (produtividade máxima da cultivar, 72,02 $\mathrm{t} \mathrm{ha}^{-1}$ ) com a aplicação da mesma quantidade de $\mathrm{N}\left(100 \mathrm{~kg} \mathrm{ha}^{-1}\right.$ de $\mathrm{N}$ ), seriam necessários cerca de $225 \mathrm{~kg}$ $\mathrm{ha}^{-1}$ de $\mathrm{K}_{2} \mathrm{O}$. Porém, a produtividade máxima poderia ser alcançada com a aplicação de $125 \mathrm{~kg} \mathrm{ha}^{-1}$ de $\mathrm{N}$ e $103 \mathrm{~kg}$ $\mathrm{ha}^{-1}$ de $\mathrm{K}_{2} \mathrm{O}$ (Figura 2C). Para a 'Superex', considerando o mesmo raciocínio, para alcançar 74,35 $\mathrm{t} \mathrm{ha}^{-1}$ de bulbos, seria suficiente a aplicação de 100 $\mathrm{kg} \mathrm{ha}^{-1}$ de $\mathrm{N}$ e $81 \mathrm{~kg} \mathrm{ha}^{-1}$ de $\mathrm{K}_{2} \mathrm{O}$. No entanto, conforme a Figura 2D, mesmo com aplicação da máxima dose de $\mathrm{K}$ estudada (225 kg ha-1 de $\mathrm{K}_{2} \mathrm{O}$ ), associado a $100 \mathrm{~kg} \mathrm{ha}^{-1}$ de $\mathrm{N}$, a produtividade máxima para a cultivar Superex $(78,91$ $\mathrm{t} \mathrm{ha}^{-1}$ ) não seria alcançada, sem alteração na quantidade aplicada de N. No entanto, com a aplicação da mesma quantidade de $\mathrm{K}$ (81 $\mathrm{kg} \mathrm{ha}^{-1}$ de $\mathrm{K}_{2} \mathrm{O}$ ) e apenas mais $20 \mathrm{~kg} \mathrm{ha}^{-1}$ de $\mathrm{N}$, a produtividade seria a máxima.

As produtividades máximas obtidas neste trabalho, de 72,02 $\mathrm{t} \mathrm{ha}^{-1}$ para a 'Optima' e 78,91 t ha-1 para a 'Superex' (Figura 2C e D). Essas produtividades foram superiores à média brasileira, que, segundo Boeing (2002), é de 15 a 17 t ha $^{-1}$ de bulbos.

Conforme as equações da superfície de resposta (Tabela 2), para uma mes- 


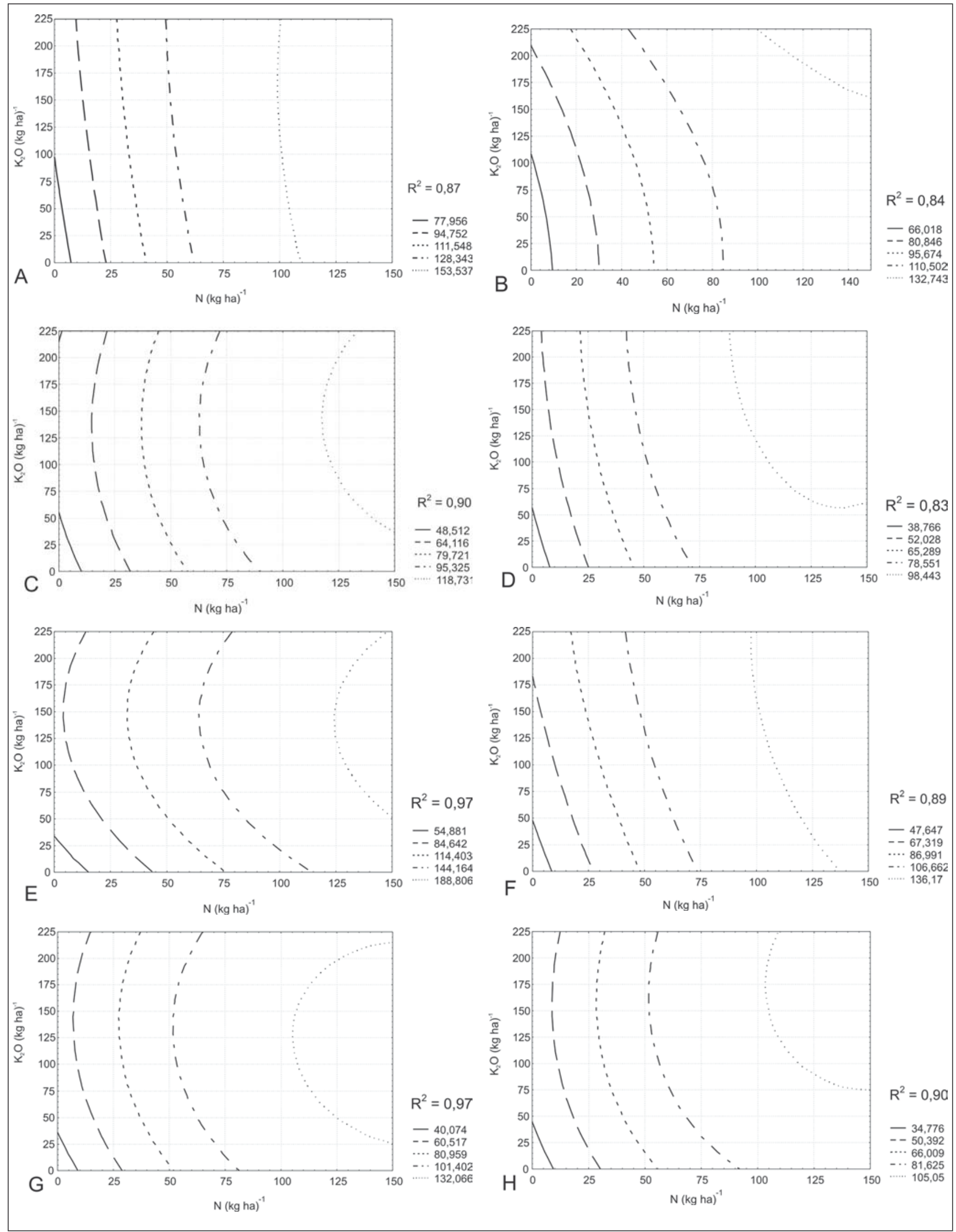

Figura 1. Isolinhas da superfície de resposta para a massa do bulbo da cultivar Optima para $60 \mathrm{pl} \mathrm{m}^{-2}(\mathrm{~A}), 76 \mathrm{pl} \mathrm{m}{ }^{-2}(\mathrm{~B}), 92 \mathrm{pl} \mathrm{m} \mathrm{m}^{-2}(\mathrm{C}), 108 \mathrm{pl}$ $\mathrm{m}^{-2}(\mathrm{D})$ e da cultivar Superex para $60 \mathrm{pl} \mathrm{m}^{-2}(\mathrm{E}), 76 \mathrm{pl} \mathrm{m}^{-2}(\mathrm{~F}), 92 \mathrm{pl} \mathrm{m}^{-2}(\mathrm{G}), 108 \mathrm{pl} \mathrm{m}^{-2}(\mathrm{H})$, em função das doses de nitrogênio e de potássio (Isolines of the answer for bulb mass of the cultivar Optima on $60 \mathrm{pl} \mathrm{m}^{-2}$ (A), $76 \mathrm{pl} \mathrm{m}^{-2}$ (B), $92 \mathrm{pl} \mathrm{m}^{-2}$ (C), $108 \mathrm{pl} \mathrm{m}$ (D) and of the cultivar Superex on $60 \mathrm{pl} \mathrm{m}^{-2}(\mathrm{E}), 76 \mathrm{pl} \mathrm{m}^{-2}(\mathrm{~F}), 92 \mathrm{pl} \mathrm{m}^{-2}(\mathrm{G}), 108 \mathrm{pl} \mathrm{m}^{-2}(\mathrm{H})$, as result of doses of $\mathrm{N}$ and K). Jaboticabal, UNESP, 2004. 

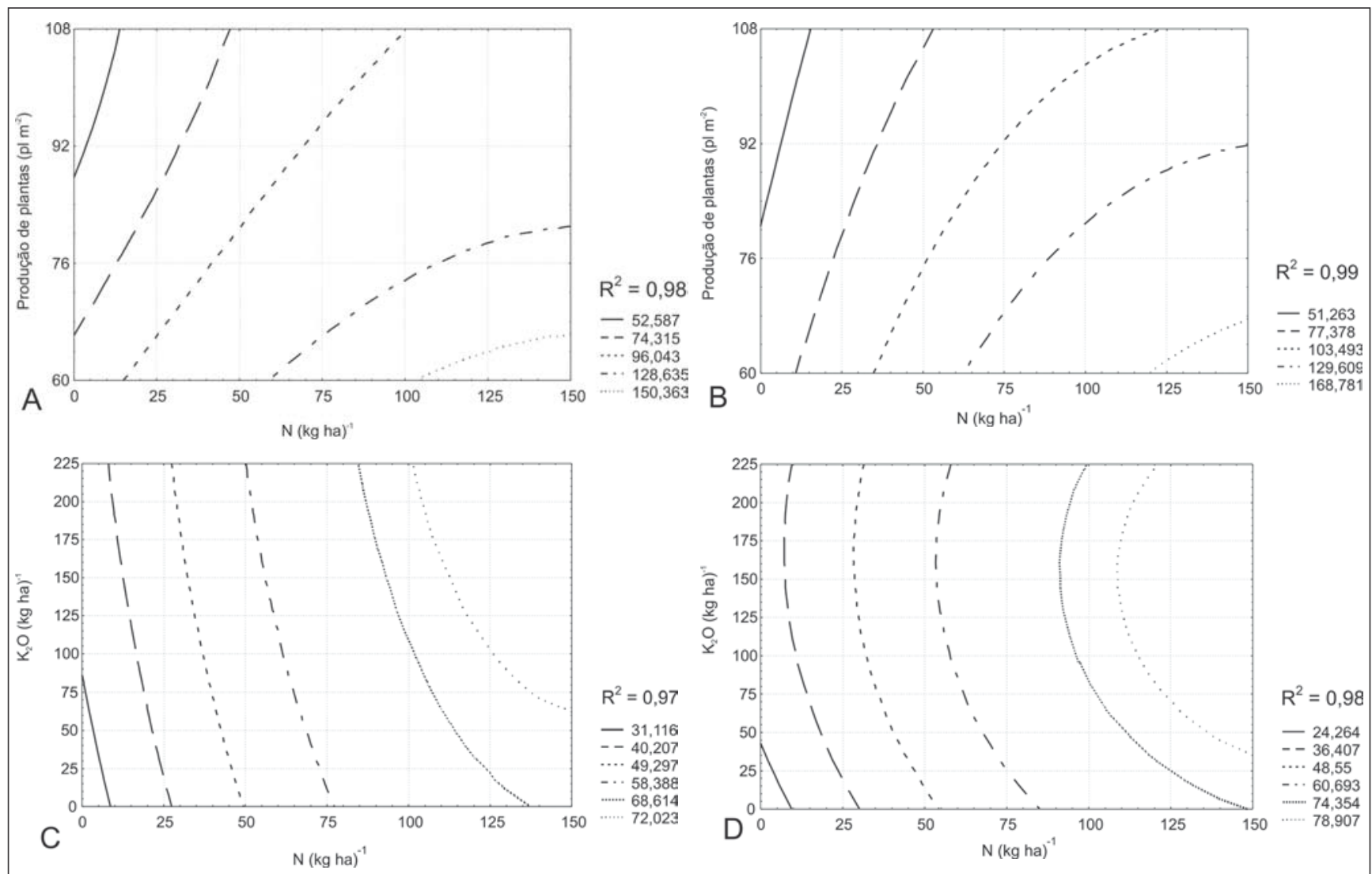

Figura 2. Isolinhas da superfície de resposta para a massa do bulbo da cultivar Optima (A) e Superex (B) em função das doses de nitrogênio e da população de plantas, e para a produtividade total da cultivar Optima (C) Superex (D) em função de doses de nitrogênio e potássio (Isolines of the surface for bulb mass from cultivar Optima (A) and Superex (B) as result of doses of N and plants population and, for total yield of cultivar optima (C) and Superes (D) as a function of doses of N and K). Jaboticabal, UNESP, 2004.

ma produtividade, existem inúmeras combinações N x K possíveis (Figura 2). Observa-se que, considerando $90 \%$ da produtividade máxima, há uma redução significativa das doses de N e K necessárias. Neste sentido, para se obter $64,8 \mathrm{t} \mathrm{ha}^{-1}$ de bulbos na cultivar Optima (90\% da produção máxima), se não for realizada a aplicação de potássio, são necessários $105 \mathrm{~kg} \mathrm{ha}^{-1}$ de $\mathrm{N}$. Para a cultivar Superex, objetivando $90 \%$ da produtividade máxima $\left(71 \mathrm{t} \mathrm{ha}^{-1}\right)$, com zero de potássio, deve-se aplicar 125 $\mathrm{kg} \mathrm{ha}^{-1}$ de N. Assim, há uma redução na necessidade de fertilizantes com pequena diminuição na produtividade almejada, além de dispensar a aplicação de $\mathrm{K}$, para as condições de fertilidade original do solo estudado, cujo teor do nutriente foi de $1,4 \mathrm{mmol}_{\mathrm{c}} \mathrm{dm}^{-3}$. Embora este teor de K no solo seja considerado baixo na proposta de adubação da cultura de Trani et al. (1996), observa-se que o incremento na produtividade da cultura em resposta ao aumento nas do- ses de $\mathrm{K}$ foi pequeno, quando comparado ao que o $\mathrm{N}$ proporcionou. As respostas da cultura a esse nutriente são, de maneira geral, pouco expressivas (Magalhães, 1993), de forma contrária ao que se observa para N (Brewster, 1994).

A população de plantas não influenciou a produtividade, e pode ser explicado pela compensação existente entre a massa do bulbo, que aumentou gradativamente com o menor número de plantas por metro quadrado. No entanto, há necessidade de fertilizações nitrogenadas em doses diferenciadas para cada situação estudada, visando alcançar a massa do bulbo comercialmente mais desejável.

Os valores preditos no ponto crítico para a 'Optima' e 'Superex' para a variável produtividade estão acima da faixa de fertilização estudada em razão do tipo de ponto crítico (máximo). Portanto, é provável que possa existir alguma resposta dessas cultivares quando fertilizadas em níveis superiores aos aqui estudados.

\section{AGRADECIMENTOS}

À FAPESP pelo auxílio à pesquisa concedido; ao CNPQ pela bolsa de doutorado; às empresas Agristar do Brasil Ltda e Takii do Brasil Ltda, nas pessoas de Maurício Coutinho Pellegrini e Eduardo Hideomi Seo, respectivamente; a Rosemari Teotônio Rodrigues; a Salete Anelise Nappi pela ajuda nos trabalhos de campo; e ao produtor rural Osmar Molina.

\section{REFERÊNCIAS}

ARAÚJO MT; COSTA RA. 1975. Efeito da adubação NPK e adubo de lixo industrializado sobre a produção de cebola (Allium cepa L.) em solos de cerrado. Revista de Olericultura 13: 111-115.

BOEING G. 2002. Fatores que afetam a qualidade da cebola na agricultura familiar catarinense. Florianópolis: Instituto CEPA/SC. $88 \mathrm{p}$.

BREWSTER JL. 1994. Onion and other vegetable Alliums. Wellesbourne: Horticulture Research International/CAB Internacional. 236 p. 
DELLACECCA V; LOVATO AFS. 2000. Effects of different plant densities and planting systems on onion (Allium cepa $\mathrm{L}$.) bulb quality and yield. Acta Horticulturae 533: 197-201.

EMBRAPA. 1999. Sistema Brasileiro de Classi ficação de Solos. Rio de Janeiro: Embrapa Solos. 412p.

FAQUIN V. 1994. Nutrição Mineral de Plantas. Lavras: ESAL/FAEPE. 227p.

FINGER FL; CASALI VWD. 2002. Colheita, cura e armazenamento da cebola. Informe Agropecuário 23: 93-98.

HENRIKSEN K. 1987. Effect of N and P fertilization on yield and harvest time in bulb onions. Acta Horticulturae 198: 207-215.

MAGALHÃES JR. 1993. Nutrição e adubação da cebola. In: FERREIRA ME; CASTELLANE PD; CRUZ MCP. (Ed.). Nutrição e adubação de hortaliças. Piracicaba: Potafos. p. 381-393.
NICHOLS MA. 1967. A note on a plant density and fertilizer experiment with onions in New Zealand. Horticultural Research 7: 144-147.

PANDE RC; MUNDRA RS. 1971. Note on response of onion (Allium cepa $\mathrm{L}$.) to varying levels of N, P and K. Indian Journal of Agricultural Science 41: 107-108.

RAIJ B. Van; ANDRADE JC; CANTARELLA H; QUAGGIO JA. 2001. Análise Química para avaliação da Fertilidade de Solos Tropicais. Campinas: Instituto Agronômico. 285p.

RUMPEL J; FELCZYNSKI K. 2000. Effect of plant density on yield and bulb size of direct sown onions. Acta Horticulturae 533: 179-186.

SANTOS HS; TANAKA MT; WATANABE SH; ARANTES PAZ; IVONE TT. 2000. Produção de cebola em função de tamanho de muda e espaçamento. Horticultura Brasileira 18: 556-557.
SILVA E; TEIXEIRA LAJ; AMADO TJC. 1991. The increase in onion production in Santa Catarina State, South Brazil. Onion Newsletter for the Tropics 3: 7-9.

STOFFELLA PJ. 1996. Planting arragement and density of transplants influence sweet spanish onion yields and bulbs size. Hortscience 7: 1129-1130.

TRANI PE; TAVARES M; SIQUEIRA WJ. 1996. Cebola (sistema de mudas). In: RAIJ B Van; CANTARELLA H; QUAGGIO JA; FURLANI AMC. Recomendações de adubação e calagem para o estado de São Paulo, Campinas/SP: IAC. p. 176. (Boletim Técnico 100).

VIDIGAL SM; PEREIRA PRG; PACHECO DD. 2002. Nutrição mineral e adubação da cebola. Informe Agropecuário 23: 36-50. 